\title{
Erratum
}

\section{Plant community dynamics in a chain of lakes: principal factors in the decline of rooted macrophytes with eutrophication}

\author{
R. Anton Hough, Mark D. Fornwall, Brian J. Negcle, Robcrt L. Thompson \& David A. Putt \\ Department of Biological Sciences, Wayne State University, Detroit, MI 48202, USA
}

Hydrobiologia 173: 199-217, 1989.

In Table 3 on page 206 of the above article, the growth habits of Najas flexilis, Myriophyllum verticillatum, $M$. spicatum, Potamogeton filiformis, and P. zosteriformis should be listed as SR (submerged rooted). The full corrected table is given below.

Table 3. Percent of all samples* from each lake containing each macrophyte species.

\begin{tabular}{|c|c|c|c|}
\hline Taxon & $\begin{array}{l}\text { Growth } \\
\text { babit }+\end{array}$ & $\begin{array}{l}\text { Shoe } \\
\text { Lake }\end{array}$ & $\begin{array}{l}\text { East Graham } \\
\text { Lake }\end{array}$ \\
\hline Ceratophyllum demersum $\mathrm{L}$. & NR & 91 & 30 \\
\hline Utricularia vulgaris L. var. americana Gray & NR & 49 & 15 \\
\hline Myriophyllum verticallatum $\mathrm{L}$. & SR & 35 & 0 \\
\hline Najas flexilis (Willd.) Rostk. and Schmidt & $\mathrm{SR}$ & 26 & 12 \\
\hline Potamogeton natans $\mathrm{L}$. & FR & 18 & 1 \\
\hline Potamogeton zosteriformis Fernald & SR & 36 & 39 \\
\hline Potamogeton filiformis Pers. & SR & 2.6 & 1 \\
\hline Nuphar variegatum Engelm. & FR & 2 & 5 \\
\hline Nymphaea tuberosa Paine & FR & 13 & 10 \\
\hline Pontederia cordata $\mathrm{L}$. & E & 1 & 2 \\
\hline Nitella flexilis (L.) Ag. & SR & 0.6 & 14 \\
\hline Elodea canadensis (Minchx.) Planchon & SR & 6 & 20 \\
\hline Potamogeton illinoensis Morong & SR & 8 & 24 \\
\hline Myriophyllum spicatum L. var. exalbescens & SR & 3 & 54 \\
\hline Chara vulgaris $\mathrm{L}$. & SR & 25 & 56 \\
\hline Vallisneria americana Michx. & $\mathrm{SR}$ & 0 & 0.3 \\
\hline Utricularia intermedia Hayne & NR & 0 & 0.3 \\
\hline Eleocharis equisetoides (Ell.) Torr. & $\mathrm{E}$ & 0 & 3 \\
\hline Brasenia schreberi (Gmel.) & FR & 0 & 4 \\
\hline Scirpus subterminalis Torr. & SR & 0 & 4 \\
\hline Potamogeton amplifolius Tuckerm. & $\mathrm{SR}$ & 0 & 11 \\
\hline Potamogeton pectinatus $\mathrm{L}$. & $\mathrm{SR}$ & 0 & 28 \\
\hline
\end{tabular}

* 180 samples from Shoe Lake; 324 samples from East Graham Lake.

+ Denotes growth habit: NR = non-rooted, $\mathrm{SR}=$ submerged rooted, $\mathrm{FR}=$ floating leaved-rooted, $\mathrm{E}=$ emergent. 